\title{
ETS transcription factors in hematopoietic stem cell development
}

\author{
Aldo Ciau-Uitz ${ }^{1 \#}$, Lu Wang ${ }^{2 \#}$, Roger Patient ${ }^{1}$ and Feng Liu ${ }^{2 *}$ \\ ${ }^{1}$ Weatherall Institute of Molecular Medicine, John Radcliffe Hospital, University of Oxford, Oxford, \\ OX3 9DS, United Kingdom
}

${ }^{2}$ State Key Laboratory of Biomembrane and Membrane Biotechnology, Institute of Zoology, Chinese Academy of Sciences, Beijing, 100101, China

\# These authors contributed equally to this work.

\section{* Corresponding author}

Feng Liu

State Key Laboratory of Biomembrane and Membrane Biotechnology

Institute of Zoology, Chinese Academy of Sciences,

Beijing, 100101, China

Email: liuf@ioz.ac.cn

Tel: +86 (10) 64807307

Fax: +86 (10) 64807313

http://www.biomembrane.ioz.ac.cn/Enliuf.asp 


\section{Abstract}

Hematopoietic stem cells (HSCs) are essential for the maintenance of the hematopoietic system. However, these cells cannot be maintained or created in vitro, and very little is known about their generation during embryogenesis. Many transcription factors (TFs) and signaling pathways play essential roles at various stages of HSC development. Members of the ETS ('E twenty-six') family of TFs have become increasingly recognized as key regulators within the gene regulatory networks (GRNs) governing hematopoiesis, including the ontogeny of HSCs. Remarkably, although all ETS TFs bind the same DNA consensus sequence and overlapping tissue expression is observed, individual ETS TFs play unique roles in the development of HSCs. Also, these TFs are recurrently used throughout development and their functions are context-dependent, increasing the challenge of studying their mechanism of action. Critically, ETS factors also play roles under pathological conditions, such as leukemia and, therefore, deciphering their mechanism of action will not only enhance our knowledge of normal hematopoiesis, but also inform protocols for their creation in vitro from pluripotent stem cells and the design of new therapeutic approaches for the treatment of malignant blood diseases. In this review, we summarize the key findings on the roles of ETS TFs in HSC development and discuss novel mechanisms by which they could control hematopoiesis.

Key words: ETS transcription factors, Hematopoietic stem cell, Hemangioblast, Endothelium, Endothelial to hematopoietic transition, Gene regulatory network, Embryo 


\section{Introduction}

Hematopoiesis, including the generation of HSCs during embryogenesis, is a complex process in which multiple regulatory pathways converge to orchestrate tissue formation, cell movement and cell fate decisions including lineage specification and differentiation [1]. Defining the molecular mechanisms underlying these processes is essential to understanding how their development takes place and is clinically relevant for regenerative medicine. It is widely accepted that TFs execute the GRNs controlling the multiple processes involved in hematopoiesis, integrating the multiple endogenous and environmental clues which influence cell behavior. Within the hematopoietic system, a number of TFs have been shown to be essential, including several members of the ETS TF family.

ETS TFs comprise a large evolutionarily conserved gene family characterized by sequence homology within their DNA binding ETS domain, which forms a winged helix-turn-helix structural motif that binds to the consensus sequence, GGAA/T, the ETS binding site found in target genes [2]. ETS TFs are involved in the regulation of a variety of biological processes and are present in all of the metazoan genomes analyzed to date [3]. Interestingly, the number of ETS TFs has increased in vertebrates compared to invertebrate metazoans: while in Drosophila and C. elegans, 8 and 10 genes, respectively, are present, in vertebrates $26+$ members of this family are found $[2 ; 3 ; 4 ; 5]$. ETS gene radiation in vertebrates might be related to the development of a sophisticated hematopoietic system and the emergence of a closed circulatory system. Although hematopoiesis takes place in Drosophila and the orthologs of well characterized vertebrate blood TFs (Runx, Gata, Fog) regulate this process, no members of the ETS family have been shown to be involved [6]. Drosophila hematopoiesis produces phagocytes which comprise the innate immune system and are analogs of vertebrate myeloid cells [7]. 
In vertebrates, the development of myeloid cells is controlled by one of the most versatile master regulators of hematopoietic fate decisions, the ETS TF PU.1 [8; 9; 10]. Strikingly, PU.1 and other closely related ETS TFs, SpiB and SpiC, have only been identified in vertebrates $[11 ; 12]$ where they control not only the development of the innate but also of the adaptive immune system [8]. Thus, it appears that new ETS TFs emerged and co-evolved with the vertebrate adaptive immune system. In vertebrates, a large number of ETS TFs are expressed and play essential roles in hematopoietic cells and the vasculature, with the GRNs controlling the development of these tissues containing ETS TFs at their core. For example, an ETS-FoxC interaction controls expression in endothelial cells [13] while an ETS-Gata interaction is at the core of hematopoietic expression [14; 15].

The ETS family comprises activators and repressors as well as proteins with both activation and repression activities [16]. In hematopoietic and endothelial cells, a number of ETS TFs are co-expressed at any single time, suggesting that a degree of functional redundancy may exist or that they act in a combinatorial manner to control development. Nevertheless, even if all ETS TFs bind the same consensus sequence and several members of the family may be present in a particular tissue at a specific time in development, a wealth of evidence indicates that individual ETS TFs play distinct, non-overlapping functions. Here, we discuss the key roles that ETS factors play in hematopoiesis, in particular in the ontogeny of the HSC.

The role of Etv2, Etv6 and Fli1 in the specification of the hematopoietic lineage from nascent mesoderm

In mammalian and avian embryos, hematopoietic specification initiates when nascent mesodermal cells 
of the primitive streak are specified as cardiovascular progenitors, some of these progenitors migrate to the yolk sac to become hemangioblasts, the presumptive common progenitor cells for blood and endothelium. Expression of Flk1/VegfR2 by Brachyury-expressing cells is thought to mark the emergence of hemangioblasts [17] and, therefore, it has been considered that Flk1 signaling initiates the GRN controlling hemangioblast development. In agreement, Flk1 deficiency in the mouse results in the absence of blood and endothelial development [18]. In the zebrafish, however, Flk1 deficiency is less significant for hemangioblast specification $[19 ; 20 ; 21]$, but the cloche mutation impairs hemangioblast development [22; 23]. Nevertheless, the identity of the gene(s) mutated in cloche has not been confirmed and therefore the precise role of this factor(s) has not been characterized in other models.

Recent studies have shown that, although in zebrafish and Xenopus blood development is not completely dependent, Etv2/Er71/Etsrp is absolutely required for blood and endothelial development in the mouse [24]. Moreover, initial analysis of Etv2-deficient mouse embryos showed that Flk1 expression is dependent on Etv2, therefore suggesting that Etv2 sits at the top of the GRN controlling the emergence of blood and endothelial progenitors [24]. In support of this, Etv2 has been shown to bind Flk1's promoter in mouse cells [24] and directly activate its expression in Xenopus embryos [25]. In addition, Flk1 expression is absent in Etv2-deficient zebrafish embryos $[5 ; 26 ; 27]$ and exogenous Etv2 can induce Flk1 expression in both fish and Xenopus, even in cloche mutants [25; 27]. However, more recently, it has been demonstrated that in ES cells Etv2 is dispensable for the generation of Flk1+ mesoderm; in Etv2-deficient ES cells, Flk1+ cells are generated but their development is arrested [28], presumably, before hemogenic endothelium is specified and, as a consequence, blood cells are not 
generated [28; 29]. Furthermore, VEGFA rapidly induces high levels of Etv2 expression in Flk1+ cells, strongly suggesting that Etv2 is downstream of Flk1 [28]. More recently, to clarify the genetic hierarchy between Flk1 and Etv2, Rasmussen et al [30] used a transgenic Etv2-EYFP reporter mouse and showed that Etv2 expression is restricted to a subset of Flk1+ cells during primitive streak stages. Furthermore, the number of Flk1-expressing cells in Etv2-deficient embryos is only modestly reduced, while Etv2-EYFP expression is almost undetectable in Flk1-deficient embryos [30]. Additionally, it was demonstrated that the Etv2 promoter is activated by VEGFA-Flk1 signaling, strongly indicating that, in the earliest hematoendothelial progenitors emerging from the primitive streak, Flk1 is required for Etv2 expression [30]. Taken together, the evidence indicates that Flk1 and Etv2 can activate each other bidirectionally in a context-dependent manner and that a feasible scenario would be that Flk1 is required for the initial expression of Etv2 but that, after hematoendothelial progenitors have been specified, Flk1 may require an Etv2 positive feedback for its expression in developing blood vessels.

All the observations presented above relate to the development of embryonic hemangioblasts which give rise to the transient yolk sac embryonic hematopoiesis. Elucidation of the epistatic relationship between Flk1 and Etv2, at the time HSCs emerge from the hemogenic endothelium associated with the ventral wall of the dorsal aorta (DA), has not been possible due to the complete absence of the DA itself in both Flk1- and Etv2-deficient embryos in all models where it has been looked at $[12 ; 18 ; 25$; 27; 31]. It has been demonstrated that HSC emergence is intimately linked to the development of the DA and hence understanding how the earliest progenitors to the DA are programmed would inherently reveal the earliest events in HSC programming. In the mouse, lineage labeling has demonstrated that all 
endothelial cells of the DA, as well as all blood cells in the bone marrow, derive from lateral plate mesoderm $[32 ; 33 ; 34]$. Nevertheless, the exact location of the DA/HSC progenitors is not known, nor if they derive from a population of adult haemangioblasts. In zebrafish embryos, the DA derives from hemangioblasts localized in the lateral plate but they are intermingled with embryonic hemangioblasts giving rise to primitive red cells [35] and therefore the programming of the adult as opposed to embryonic hemangioblasts cannot be analyzed easily. As in the mouse, in Xenopus embryos the DA and adult hematopoiesis derive from lateral plate mesoderm $[36 ; 37 ; 38]$. The dorsal lateral plate (DLP) mesoderm of Xenopus embryos contains a population of adult hemangioblasts which, in contrast to the zebrafish, have a distinct origin and anatomical location to that of the embryonic hemangioblasts and therefore we have exploited these advantages to study the earliest programming of the HSC lineage [31; $38 ; 39 ; 40]$. We have established that adult hemangioblast programming requires synergism between a VEGFA-dependent and a VEGFA-independent pathway, controlled by the ETS TFs, Etv6/Tel1 and Fli1, respectively (Figure 1; [31]). The first synergistic interaction between these pathways takes place when VEGFA produced in the somites, under the control of Etv6, activates Flk1+ cells in the DLP itself where Fli1 together with Gata2 ensure the expression of Flk1 (Figure 1A); as a result, an endogenous, cell autonomous Etv6-VEGFA circuit is established. Simultaneously, in a VEGFA-independent manner, Fli1 and Gata2 activate the transcription of Etv2 (Figure 1B) which in its turn activates Lmo2 and maintains Gata2 expression to set the second synergistic interaction between the VEGFA-dependent and VEGFA-independent pathways to ultimately activate Scl (Figure 1C; [31; 41]). Thus, during adult hemangioblast specification, Flk1 and Etv2 transcriptional control is mutually independent. The adult hemangioblast GRN also shows that two ETS TFs are at the top of the hierarchy, Etv6 and Fli1. Evidence in the zebrafish also indicates that Fli1 does not lie downstream of Etv2 since Fli1-expressing 
cells in the posterior lateral plate mesoderm can be detected in Etv2-deficient embryos [27]. The relationship between Etv6 and Etv2 has not been investigated in the mouse or any other system. Nevertheless, the adult hemangioblast GRN shows that ETS TFs, despite sharing the same DNA binding consensus sequence, play essential and unique functions in the establishment of the HSC lineage.

Using the ES cell differentiation system, it was initially demonstrated that haemangioblasts and cardiac cells derive from Flk1-expressing progenitors [42]. More recently, it has been demonstrated in embryos that haemangioblasts and cardiac cells are indeed intimately related $[43 ; 44 ; 45 ; 46]$. In zebrafish embryos, embryonic haemangioblasts and cardiac cells derive from a common population of mesodermal progenitors; within this population, an Fgf signaling regime dictates cardiogenic vs haemangioblast fate, favoring cardiac differentiation [44]. Moreover, a cross antagonism between haemangioblast and cardiac TFs has been demonstrated, in other words, haemangioblast TFs repress the cardiac fate whereas cardiac TFs repress the haemangioblast fate [43; 44; 45; 46]. More recently, Etv2 has emerged as a key regulator of haemangioblast vs cardiac fate determination. Unlike Fli1, Gata2 and Flk1, whose expression persists after hemangioblast specification, Etv2 expression in ES cells is transient, taking place only between Days 1-4 of differentiation [24; 29]. Interestingly, Flk1 negative mesoderm isolated at Day 3.5 of differentiation becomes Flk1+ by Day 4.25 [42], presumably in the absence of Etv2, further indicating that nascent cardiovascular progenitors do not require Etv2 to activate Flk1. This second wave of Flk1+ cells gives rise to cardiac progenitors [42]. Furthermore, in the zebrafish it has been shown that Etv2 is actually required to repress the cardiac fate in the anterior 
lateral plate mesoderm while favoring hematopoietic specification [47]. Taken together, Etv2 appears to modulate fate decisions in nascent cardiovascular progenitors where, on the one hand, it instructs a subpopulation of Flk1+ cells, very likely through activation of Scl [45; 48] or repression of Wnt signaling [49], to acquire the hematoendothelial fate while repressing their cardiac potential and, on the other hand, Flk1+ cells need to be released from Etv2 activity to express their cardiac potential (Figure 2).

\section{ETS TFs and vascular development}

The emergence of HSCs is intimately associated with blood vessel endothelium. In fact, HSCs are generated from the endothelium of the major arteries of the developing embryo [50]. In particular, it has been shown that the first HSCs emerge in association with the ventral wall of the lumenised and fully functional DA (see accompanying reviews in this issue). Therefore, understanding how the DA is specified would inform on the mechanism by which HSCs are programmed. Deficiencies of most of the key hematopoietic TFs have an impact on hemangioblast specification which results in early embryo lethality in the mouse due to defects in primitive blood and blood vessel development, and as a consequence hematopoiesis in the DA cannot be analyzed. In contrast, fish and frog embryos are less dependent on oxygen delivered by the circulation and embryos deficient in the same TFs survive long enough to analyze the emergence of HSCs in the DA. Thus, the zebrafish and Xenopus models have greatly contributed to our understanding of the development of the DA/HSC precursors [51]. Using these models, it has been shown that the DA and HSCs derive from hemangioblasts arising in the lateral plate mesoderm, then a subpopulation of these cells migrate to the midline where they coalesce to form the DA. Subsequently, the DA polarizes and hemogenic endothelium is specified on its ventral 
aspect where HSCs eventually emerge [51].

ETS TFs also feature prominently in the GRN controlling the development of the vascular system, including the lymphatic vessels [52]. Central to this GRN is a conserved Fox:ETS interaction [13; 52] whereby, to drive endothelial expression, ETS factors function in cooperation with FoxC TFs via a composite element, the FOX:ETS motif [13; 53]. FoxC2 and Etv2 can bind this motif simultaneously and activate all of the enhancers containing this motif [13]. Thus, coexpression of FoxC2 and Etv2 causes ectopic expression of endothelial genes in Xenopus embryos, while the combined knockdown of the orthologous genes in zebrafish embryos induces vascular defects [13]. However, although Etv2 and FoxC2 were originally reported to transactivate expression in endothelial cells, evidence suggests that other ETS and Fox TFs may utilize the Fox:ETS motif to control endothelial development and or maintenance.

A number of ETS TFs are expressed in the developing and mature vasculature but only a few appear to impact vascular integrity. Apart from Etv2, the deficiency of single ETS TFs appears to have little impact on blood vessel development [54]. For example, Ets1, the founding member of the ETS family, is highly expressed in embryonic endothelial cells and can directly regulate many specific vascular genes [55; 56]; nevertheless, in ets1-deficient zebrafish embryos, endothelial cells are still present and circulation is only lost after $24 \mathrm{hpf}$ [57]. Fli1 is also highly expressed in the endothelial lineage and null mutations in the mouse results in lethality at E12.5 due to disrupted blood vessel integrity [58], but its deficiency in the zebrafish only displays a partial loss of trunk circulation and hemorrhage in the head [5]. Similarly, Elk3, a vascular specific ETS TF, does not appear to be essential for vascular 
development [59]. This suggests that, within the vasculature, ETS TFs play redundant roles or that they are not involved in vascular integrity but rather other vascular functions. Indeed, Elk3 has been shown to be required for lymphatic vessel integrity [59] and, in Etv6-deficient embryos, vascular development takes place normally but arterial specification of the DA fails and, subsequently, hemogenic endothelium/HSC programming fails [41].

\section{The potential role of ETS factors in the endothelial to hematopoietic transition (EHT)}

The emergence of the first HSCs in the ventral wall of the DA is thought to be by a novel process termed the endothelial to hematopoietic transition (EHT)([60] and accompanying reviews in this issue).

The runt domain TF, Runx1, plays a key role in both the specification of hemogenic endothelium and EHT but the mechanism by which it controls these processes is currently unknown. EHT superficially resembles the widely observed epithelial to mesenchymal transition (EMT) and Runx factors have recently been shown to regulate EMT in malignant tissues [61]. Runx1, Runx2 and Runx3 are expressed in thyroid carcinomas, and analysis of Runx2 function demonstrated that expression of angiogenic factors, such as VEGFA and VEGFC, as well as EMT-related factors, such as Snail2, Snail3 and Twist1, are regulated by Runx2 [61]. Thus, during HSC emergence, it is possible that Runx1 controls EHT by regulating EMT-related factors.

A number of ETS TFs have been shown to play key roles in EMT in malignant tissues. On the one hand, Ets1 in renal epithelial cells [62], Elf5, Erf and Pdef in breast cancer cells [63; 64; 65], Ese3 in prostate cancer [66], and Etv5 in ovarian cancer cells [67] repress EMT. On the other hand, Erg in 
prostate cancer cells [68] and Etv4 in prostate, lung, ovarian and breast cancer cells [69; 70], induce EMT. EMT regulation in these malignant tissues generally involves the transcriptional regulation of key EMT TFs such as Snail1, Snail2, Twist1, Zeb1 and Zeb2; for example, Ets1 binds the promoter of Zeb1 and directly regulates its expression [71], while Etv4 regulates the expression of Twist1, Snail1, Zeb1 and Zeb2 [69]. Therefore, if EMT and EHT share a common molecular machinery, due to their expression in hemogenic endothelium, ETS TFs are very likely to be involved in the regulation of EHT during the emergence of HSC from hemogenic endothelium.

Runx1 and ETS TFs have been shown to interact at multiple levels. ETS TFs might be involved in Runx1 transcriptional regulation since a Runx1 enhancer, which drives its expression in emerging HSCs, contains ETS binding sites required for its activity and this enhancer can be bound by Fli1, Elf1 and Pu.1 [72]; reciprocally, Runx1 regulates the expression of hematopoietic ETS TFs [15; 73]. Also, genome wide ChIPseq data indicate that Runx1 and ETS TFs co-regulate a large cohort of hematopoietic genes [15; 74]. Importantly, Runx1 can physically bind ETS TFs [75; 76; 77; 78]. It has been shown that the Runx1 physical interaction with ETS TFs is conserved in Drosophila, where Lozenge and Pointed, the orthologs of Runx1 and Ets1/Ets2 respectively, associate before DNA binding [78]. It was also shown that Runx1 interaction with ETS TFs is independent of the runt domain, it is the Lozenge ExonV in Drosophila and Runx1 ExonVI in mammals, which physically associates with the pointed domain of ETS TFs [78]. Therefore, any ETS TF containing the pointed domain (Ets1, Ets2, Fli1, Erg, Gabpa, Elf3, Elf5, Ese3, Pdef, Etv6 and Etv7) is capable of physically associating with Runx1 and potentially co-regulating the EHT and other processes during HSC generation. Of the ETS TFs containing the pointed domain, Ets1, Erg, Elf5, Ese3 and Pdef have been shown to regulate EMT 
in cancer cells and therefore, if expressed in hemogenic endothelium, could drive EHT together with Runx1. Thus, ETS TFs are very likely to be at the core of the EHT modulated by Runx1 and the generation of the first HSCs.

\section{HSC specification and initiation}

A number of TFs that play essential roles in HSC emergence are regulated by ETS TFs [79]. For example, Scl is important for the establishment of the hemogenic endothelium in mouse embryos [80] and, in zebrafish, marks and is required for hemangioblast specification. Thus, its deficiency results in both hematopoietic and endothelial development defects, which lead to the absence of the DA and definitive hematopoiesis $[81 ; 82]$. Expression of $s c l$ in hemangioblasts and HSCs is regulated by an enhancer bound by a Gata2 and ETS TF (Fli1, Elf1) protein complex [14]. Gata2, Fli1 and Scl have been shown to form a transcriptional complex on enhancers containing Gata, ETS and E-box binding motifs, formation of this protein complex drives expression of its targets specifically in hemangioblasts and HSCs [83]. Moreover, these TFs contain those DNA binding motifs in their own regulatory elements and co-regulate each other to form a self-regulating transcriptional circuit which maintains their expression in emerging HSCs [83]. As discussed above, Runx1 is essential in the EHT process generating HSCs in the DA [84] and in vivo studies show that it can be directly regulated by GATA2 and ETS TFs. A Runx1 intronic enhancer containing Gata, ETS and E-box binding sites can recruit GATA2, Fli1, Elf1, Pu.1, and Scl in vitro, and in vivo experiments have shown that this enhancer is sufficient to drive expression in emerging HSCs, and therefore that it is responsible for Runx 1 expression in these cells [72]. 
The ETS TF, Fev (also known as Pet1), which was originally identified in serotonergic neurons in zebrafish and mammals [85; 86; 87], is also expressed in blood/endothelial cells in zebrafish [5]. More recently, we have demonstrated that Fev deficiency results in a reduction of Runx1 expression in the $\mathrm{DA}$ and fewer $\mathrm{T}$ cells in the thymus, and that Fev regulates the number of Runx1 expressing cells by transcriptionally activating its target, Erk2, to enhance ERK signaling [88]. Thus, Fev is a novel ETS TF involved in the genesis of HSCs.

\section{ETS TFs, miRNAs and hematopoiesis}

In the last few years, it has become evident that non-coding RNAs, in particular microRNAs (miRNAs), play important roles in normal and malignant tissues and, therefore, that their functions should be considered if we are to accurately understand developmental processes, in other words that miRNAs are an integral part of GRNs. miRNAs are a class of small non-coding RNAs that regulate gene expression by acting at the post-transcriptional level to repress multiple target genes via inhibition of translation or destabilization of mRNA [89]. miRNAs have been shown to be abundant during HSC differentiation in the bone marrow, and they appear to play key roles in HSC homeostasis, including HSC quiescence, self-renewal and ultimately fate decisions and differentiation (Reviewed in [90]). Moreover, increasing evidence indicates that deregulation of miRNA expression is involved in the pathogenesis of leukemia since they can act as oncogenes and tumor suppressors [91; 92]. Although the role of miRNAs in normal and pathological adult hematopoiesis is widely recognized, their role during developmental hematopoiesis is poorly known. 
Very little is known about the regulation of miRNAs generally. Nevertheless, ETS TFs appear to have a significant role in the regulation of hematopoietic and endothelial miRNAs. Primitive erythropoiesis in the yolk sac and differentiating ES cells initiates with the emergence of progenitors capable of giving rise to erythrocyte colony-forming cells (EryP-CFCs), these progenitors are generated transiently and miR-126 has been demonstrated to stop the development of EryP-CFCs [93]. Although some of the downstream targets of miR-126 in this process have been identified, nothing is known about its own regulation. However, in endothelial cells, miR-126 expression is regulated by Ets1 and Ets2 [94]. Likewise for zebrafish miR-142a-3p, which has been demonstrated to regulate vascular development and integrity, its targets in these particular processes have been identified but its regulation is unknown [95]. miR142 is of particular interest because it is one of the most abundantly expressed miRNAs in blood cells and it appears to be a blood-specific miRNA [96]. In agreement, zebrafish miR-142-3p influences the differentiation of primitive erythrocytes but its mechanism of action has not been defined [97]. Importantly, the promoter of miR-142 contains an essential ETS binding site which in primary bone marrow derived dendritic cells, as well as cultured macrophages, is bound and activated by PU.1 [98]; in this context, PU.1 synergizes with Runx1 and C/EBP $\beta$ to confer hematopoietic specific expression [98]. PU.1 has also been demonstrated to directly regulate at least 4 other miRNAs (miR-146a, miR-342, miR-338 and miR-155) that are required for macrophage differentiation [99]. This indicates that PU.1's function in myeloid differentiation is partly modulated by miRNAs and opens the possibility that PU.1's influence in acute myeloid leukemia (AML) may be modulated by miRNAs such as miR-142-3p [100]. In the same vein, it has recently been reported that PU.1, together with Fli1, directly activate miR-17-92 (also known as Fli3) in friend erythroleukemia and, in its turn, 
miR-17-92 regulates cell proliferation [101]. In the same system, Fli1 represses the expression of miR-17 and miR-20a, two miRNAs involved in cell proliferation arrest [101]. PU.1 has also been demonstrated to indirectly repress the expression of miR-17-92 during macrophage differentiation by inducing the expression of Egr2 which directly represses miR-17-92 [102]. Thus, ETS TFs are capable of controlling miRNA expression both positively and negatively to modulate normal hematopoiesis and malignant cell progression.

miRNAs have been shown to influence endothelial and hematopoietic differentiation in some cases by regulating ETS TF expression. For example, in endothelial cells, both miR-199a-5p and miR-200b target Ets1 to block angiogenesis [103; 104]. miR-155, a miRNA highly expressed in human cord blood CD34+ hematopoietic progenitor cells (HPCs) but lowly expressed in megakaryocytes, blocks megakaryocyte differentiation when induced in HPCs while its down regulation enhances their differentiation [105]. Further analysis indicated that Ets1, a key factor in megakaryocyte differentiation, is targeted by miR-155 [105]. miRNAs also have the capacity to control ETS TF expression in malignant blood cells. For instance, AML patients with NPM1 mutations have higher levels of miR-196a and miR-196b that regulate Erg, an adverse prognostic factor in patients with AML and T-ALL [106].

In conclusion, the epistatic relationship between ETS TFs and miRNAs constitutes an integral part of normal and pathological GRNs, and research in this field may result in novel strategies to produce blood cells, including HSCs, in vitro as well as for designing new therapies to treat malignant blood diseases. 


\section{Conclusion and Perspectives}

The hematopoietic system serves as a paradigm of development and stem cell biology and exemplifies how TFs, signaling molecules and new regulators, such as miRNAs, integrate into GRNs controlling multiple aspects of development. It appears that ETS TFs are at the core of the GRNs controlling the key aspects of hematopoietic specification during embryogenesis as well as the maintenance and differentiation of HSCs (Figure 3). The importance of this family of TFs is patent in their direct involvement in the pathology of blood diseases. Although all ETS TFs control gene expression by binding to the same consensus sequence, it is clear that they exhibit essential, non-overlapping functions and therefore deciphering how these unique functions are achieved is a major challenge for the understanding of normal and pathological hematopoiesis. Addressing how ETS TFs interact with other TFs, such as Runx1, signaling pathways and miRNAs is also a major challenge. In addition, evidence indicates that the regulation and function of single ETS TFs, such as PU.1, is context dependent and therefore that their functions must be studied in a tissue-specific manner. Thus, it is clear that a multidisciplinary approach is required to undertake these challenges. Undoubtedly, the study of ETS TFs will contribute to a better understanding of developmental hematopoiesis and therefore will help to instruct protocols for the differentiation of HSC towards particular blood lineages as well as the generation of HSCs in vitro and, ultimately, therapies for the treatment of malignant blood diseases and regenerative medicine. 


\section{Acknowledgements}

We thank Dr. Yuxia Jiao for critical reading of the manuscript. This work was supported by grants from the National Basic Research Program of China (2010CB945300, 2011CB943900), the National Natural Science Foundation of China (30971678), the Strategic Priority Research Program of the CAS (XDA01010110), and the UK Medical Research Council. 


\section{References}

[1] C. Zhang, R. Patient, F. Liu, Hematopoietic stem cell development and regulatory signaling in zebrafish, Biochim Biophys Acta, 2013, pp. 2370-4.

[2] A.D. Sharrocks, The ETS-domain transcription factor family. Nature reviews. Molecular cell biology 2 (2001) 827-37.

[3] A.H. Hart, R. Reventar, A. Bernstein, Genetic analysis of ETS genes in C. elegans. Oncogene 19 (2000) 6400-8.

[4] T. Hsu, R.A. Schulz, Sequence and functional properties of Ets genes in the model organism Drosophila. Oncogene 19 (2000) 6409-16.

[5] F. Liu, R. Patient, Genome-wide analysis of the zebrafish ETS family identifies three genes required for hemangioblast differentiation or angiogenesis. Circulation research 103 (2008) 1147-54.

[6] L. Waltzer, V. Gobert, D. Osman, M. Haenlin, Transcription factor interplay during Drosophila haematopoiesis. The International journal of developmental biology 54 (2010) 1107-15.

[7] J. Krzemien, M. Crozatier, A. Vincent, Ontogeny of the Drosophila larval hematopoietic organ, hemocyte homeostasis and the dedicated cellular immune response to parasitism. The International journal of developmental biology 54 (2010) 1117-25.

[8] S. Carotta, L. Wu, S.L. Nutt, Surprising new roles for PU.1 in the adaptive immune response. Immunological reviews 238 (2010) 63-75.

[9] R. Monteiro, C. Pouget, R. Patient, The gata1/pu.1 lineage fate paradigm varies between blood populations and is modulated by tif1gamma. The EMBO journal 30 (2011) 1093-103.

[10] M.M. Del Real, E.V. Rothenberg, Architecture of a lymphomyeloid developmental switch controlled by PU.1, Notch and Gata3. Development 140 (2013) 1207-19.

[11] S. Shintani, J. Terzic, A. Sato, et al., Do lampreys have lymphocytes? The Spi evidence. Proceedings of the National Academy of Sciences of the United States of America 97 (2000) 7417-22.

[12] M.K. Anderson, X. Sun, A.L. Miracle, G.W. Litman, E.V. Rothenberg, Evolution of hematopoiesis: Three members of the PU.1 transcription factor family in a cartilaginous fish, Raja eglanteria. Proceedings of the National Academy of Sciences of the United States of America 98 (2001) 553-8.

[13] S. De Val, N.C. Chi, S.M. Meadows, et al., Combinatorial regulation of endothelial gene expression by ets and forkhead transcription factors. Cell 135 (2008) 1053-64.

[14] B. Gottgens, A. Nastos, S. Kinston, et al., Establishing the transcriptional programme for blood: the SCL stem cell enhancer is regulated by a multiprotein complex containing Ets and GATA factors. The EMBO journal 21 (2002) 3039-50.

[15] N.K. Wilson, S.D. Foster, X. Wang, et al., Combinatorial transcriptional control in blood stem/progenitor cells: genome-wide analysis of ten major transcriptional regulators. Cell stem cell 7 (2010) 532-44.

[16] E. Lelievre, F. Lionneton, F. Soncin, B. Vandenbunder, The Ets family contains transcriptional activators and repressors involved in angiogenesis. Int J Biochem Cell Biol 33 (2001) 391-407.

[17] H.J. Fehling, G. Lacaud, A. Kubo, et al., Tracking mesoderm induction and its specification to the hemangioblast during embryonic stem cell differentiation. Development 130 (2003) 4217-27. 
[18] F. Shalaby, J. Rossant, T.P. Yamaguchi, et al., Failure of blood-island formation and vasculogenesis in Flk-1-deficient mice. Nature 376 (1995) 62-6.

[19] H. Habeck, J. Odenthal, B. Walderich, H. Maischein, S. Schulte-Merker, Analysis of a zebrafish VEGF receptor mutant reveals specific disruption of angiogenesis. Current biology : CB 12 (2002) 1405-12.

[20] L.D. Covassin, J.A. Villefranc, M.C. Kacergis, B.M. Weinstein, N.D. Lawson, Distinct genetic interactions between multiple Vegf receptors are required for development of different blood vessel types in zebrafish. Proceedings of the National Academy of Sciences of the United States of America 103 (2006) 6554-9.

[21] N. Bahary, K. Goishi, C. Stuckenholz, et al., Duplicate VegfA genes and orthologues of the KDR receptor tyrosine kinase family mediate vascular development in the zebrafish. Blood 110 (2007) 3627-36.

[22] D.Y. Stainier, B.M. Weinstein, H.W. Detrich, 3rd, L.I. Zon, M.C. Fishman, Cloche, an early acting zebrafish gene, is required by both the endothelial and hematopoietic lineages. Development 121 (1995) 3141-50.

[23] E.C. Liao, B.H. Paw, A.C. Oates, et al., SCL/Tal-1 transcription factor acts downstream of cloche to specify hematopoietic and vascular progenitors in zebrafish. Genes \& development 12 (1998) 621-6.

[24] D. Lee, C. Park, H. Lee, et al., ER71 acts downstream of BMP, Notch, and Wnt signaling in blood and vessel progenitor specification. Cell stem cell 2 (2008) 497-507.

[25] M.C. Salanga, S.M. Meadows, C.T. Myers, P.A. Krieg, ETS family protein ETV2 is required for initiation of the endothelial lineage but not the hematopoietic lineage in the Xenopus embryo. Developmental dynamics : an official publication of the American Association of Anatomists 239 (2010) 1178-87.

[26] S. Sumanas, G. Gomez, Y. Zhao, et al., Interplay among Etsrp/ER71, Scl, and Alk8 signaling controls endothelial and myeloid cell formation. Blood 111 (2008) 4500-10.

[27] S. Sumanas, S. Lin, Ets1-related protein is a key regulator of vasculogenesis in zebrafish. PLoS biology 4 (2006) e10.

[28] H. Kataoka, M. Hayashi, R. Nakagawa, et al., Etv2/ER71 induces vascular mesoderm from Flk1+PDGFRalpha+ primitive mesoderm. Blood 118 (2011) 6975-86.

[29] S. Wareing, A. Mazan, S. Pearson, et al., The Flk1-Cre-mediated deletion of ETV2 defines its narrow temporal requirement during embryonic hematopoietic development. Stem cells 30 (2012) 1521-31.

[30] T.L. Rasmussen, X. Shi, A. Wallis, et al., VEGF/Flk1 signaling cascade transactivates Etv2 gene expression. PloS one 7 (2012) e50103.

[31] A. Ciau-Uitz, P. Pinheiro, A. Kirmizitas, J. Zuo, R. Patient, VEGFA-dependent and -independent pathways synergise to drive $\mathrm{Scl}$ expression and initiate programming of the blood stem cell lineage in Xenopus. Development 140 (2013) In Press.

[32] P. Wasteson, B.R. Johansson, T. Jukkola, et al., Developmental origin of smooth muscle cells in the descending aorta in mice. Development 135 (2008) 1823-32.

[33] A.C. Zovein, K.A. Turlo, R.M. Ponec, et al., Vascular remodeling of the vitelline artery initiates extravascular emergence of hematopoietic clusters. Blood 116 (2010) 3435-44.

[34] H. Kataoka, M. Hayashi, K. Kobayashi, et al., Region-specific Etv2 ablation revealed the critical origin of hemogenic capacity from Hox6-positive caudal-lateral primitive mesoderm. 
Experimental hematology (2013).

[35] M. Gering, A.R. Rodaway, B. Gottgens, R.K. Patient, A.R. Green, The SCL gene specifies haemangioblast development from early mesoderm. The EMBO journal 17 (1998) 4029-45.

[36] M. Maeno, A. Todate, C. Katagiri, The Localization of Precursor Cells for Larval and Adult Hematopoietic-Cells of Xenopus-Laevis in 2 Regions of Embryos. Development Growth \& Differentiation 27 (1985) 137-148.

[37] O. Cleaver, P.A. Krieg, VEGF mediates angioblast migration during development of the dorsal aorta in Xenopus. Development 125 (1998) 3905-3914.

[38] A. Ciau-Uitz, M. Walmsley, R. Patient, Distinct origins of adult and embryonic blood in Xenopus. Cell 102 (2000) 787-796.

[39] M. Walmsley, A. Ciau-Uitz, R. Patient, Adult and embryonic blood and endothelium derive from distinct precursor populations which are differentially programmed by BMP in Xenopus. Development 129 (2002) 5683-5695.

[40] F. Liu, M. Walmsley, A. Rodaway, R. Patient, Fli1 acts at the top of the transcriptional network driving blood and endothelial development. Current Biology 18 (2008) 1234-1240.

[41] A. Ciau-Uitz, P. Pinheiro, R. Gupta, T. Enver, R. Patient, Tel1/ETV6 Specifies Blood Stem Cells through the Agency of VEGF Signaling. Developmental cell 18 (2010) 569-578.

[42] S.J. Kattman, T.L. Huber, G.M. Keller, Multipotent flk-1+ cardiovascular progenitor cells give rise to the cardiomyocyte, endothelial, and vascular smooth muscle lineages. Developmental cell 11 (2006) 723-32.

[43] T. Peterkin, A. Gibson, R. Patient, Common genetic control of haemangioblast and cardiac development in zebrafish. Development 136 (2009) 1465-74.

[44] F.C. Simoes, T. Peterkin, R. Patient, Fgf differentially controls cross-antagonism between cardiac and haemangioblast regulators. Development 138 (2011) 3235-45.

[45] M. Gering, Y. Yamada, T.H. Rabbitts, R.K. Patient, Lmo2 and Scl/Tal1 convert non-axial mesoderm into haemangioblasts which differentiate into endothelial cells in the absence of Gata1. Development 130 (2003) 6187-99.

[46] J.J. Schoenebeck, B.R. Keegan, D. Yelon, Vessel and blood specification override cardiac potential in anterior mesoderm. Developmental cell 13 (2007) 254-67.

[47] S. Palencia-Desai, V. Kohli, J. Kang, et al., Vascular endothelial and endocardial progenitors differentiate as cardiomyocytes in the absence of Etsrp/Etv2 function. Development 138 (2011) 4721-32.

[48] B. Van Handel, A. Montel-Hagen, R. Sasidharan, et al., Scl represses cardiomyogenesis in prospective hemogenic endothelium and endocardium. Cell 150 (2012) 590-605.

[49] F. Liu, I. Kang, C. Park, et al., ER71 specifies Flk-1+ hemangiogenic mesoderm by inhibiting cardiac mesoderm and Wnt signaling. Blood 119 (2012) 3295-305.

[50] M.F. de Bruijn, N.A. Speck, M.C. Peeters, E. Dzierzak, Definitive hematopoietic stem cells first develop within the major arterial regions of the mouse embryo. The EMBO journal 19 (2000) 2465-74.

[51] A. Ciau-Uitz, F. Liu, R. Patient, Genetic control of hematopoietic development in Xenopus and zebrafish. International Journal of Developmental Biology 54 (2010) 1139-1149.

[52] S. De Val, Key transcriptional regulators of early vascular development. Arteriosclerosis, thrombosis, and vascular biology 31 (2011) 1469-75.

[53] A.M. Randi, A. Sperone, N.H. Dryden, G.M. Birdsey, Regulation of angiogenesis by ETS 
transcription factors. Biochemical Society transactions 37 (2009) 1248-53.

[54] S.M. Meadows, C.T. Myers, P.A. Krieg, Regulation of endothelial cell development by ETS transcription factors. Seminars in cell \& developmental biology 22 (2011) 976-84.

[55] E. Lelievre, V. Mattot, P. Huber, B. Vandenbunder, F. Soncin, ETS1 lowers capillary endothelial cell density at confluence and induces the expression of VE-cadherin. Oncogene 19 (2000) 2438-46.

[56] J. Dittmer, The biology of the Ets1 proto-oncogene. Molecular cancer 2 (2003) 29.

[57] V.N. Pham, N.D. Lawson, J.W. Mugford, et al., Combinatorial function of ETS transcription factors in the developing vasculature. Developmental biology 303 (2007) 772-83.

[58] D.D. Spyropoulos, P.N. Pharr, K.R. Lavenburg, et al., Hemorrhage, impaired hematopoiesis, and lethality in mouse embryos carrying a targeted disruption of the Fli1 transcription factor. Molecular and cellular biology 20 (2000) 5643-52.

[59] A. Ayadi, H. Zheng, P. Sobieszczuk, et al., Net-targeted mutant mice develop a vascular phenotype and up-regulate egr-1. The EMBO journal 20 (2001) 5139-52.

[60] K. Kissa, P. Herbomel, Blood stem cells emerge from aortic endothelium by a novel type of cell transition. Nature 464 (2010) 112-5.

[61] D.F. Niu, T. Kondo, T. Nakazawa, et al., Transcription factor Runx2 is a regulator of epithelial-mesenchymal transition and invasion in thyroid carcinomas. Laboratory investigation; a journal of technical methods and pathology 92 (2012) 1181-90.

[62] K. Okano, A. Hibi, T. Miyaoka, et al., Inhibitory effects of the transcription factor Ets-1 on the expression of type I collagen in TGF-beta1-stimulated renal epithelial cells. Molecular and cellular biochemistry 369 (2012) 247-54.

[63] R. Chakrabarti, J. Hwang, M. Andres Blanco, et al., Elf5 inhibits the epithelial-mesenchymal transition in mammary gland development and breast cancer metastasis by transcriptionally repressing Snail2. Nature cell biology 14 (2012) 1212-22.

[64] M. Allegra, A. Zaragkoulias, E. Vorgia, et al., Semaphorin-7a reverses the ERF-induced inhibition of EMT in Ras-dependent mouse mammary epithelial cells. Molecular biology of the cell 23 (2012) 3873-81.

[65] D.P. Turner, V.J. Findlay, O. Moussa, et al., Mechanisms and functional consequences of PDEF protein expression loss during prostate cancer progression. The Prostate 71 (2011) 1723-35.

[66] D. Albino, N. Longoni, L. Curti, et al., ESE3/EHF controls epithelial cell differentiation and its loss leads to prostate tumors with mesenchymal and stem-like features. Cancer research 72 (2012) 2889-900.

[67] M. Llaurado, M. Abal, J. Castellvi, et al., ETV5 transcription factor is overexpressed in ovarian cancer and regulates cell adhesion in ovarian cancer cells. International journal of cancer. Journal international du cancer 130 (2012) 1532-43.

[68] D.D. Becker-Santos, Y. Guo, M. Ghaffari, et al., Integrin-linked kinase as a target for ERG-mediated invasive properties in prostate cancer models. Carcinogenesis 33 (2012) 2558-67.

[69] A. Pellecchia, C. Pescucci, E. De Lorenzo, et al., Overexpression of ETV4 is oncogenic in prostate cells through promotion of both cell proliferation and epithelial to mesenchymal transition. Oncogenesis 1 (2012) e20.

[70] H.F. Yuen, Y.K. Chan, C. Grills, et al., Polyomavirus enhancer activator 3 protein promotes breast cancer metastatic progression through Snail-induced epithelial-mesenchymal transition. The 
Journal of pathology 224 (2011) 78-89.

[71] N. Dave, S. Guaita-Esteruelas, S. Gutarra, et al., Functional cooperation between Snaill and twist in the regulation of ZEB1 expression during epithelial to mesenchymal transition. The Journal of biological chemistry 286 (2011) 12024-32.

[72] W.T. Nottingham, A. Jarratt, M. Burgess, et al., Runx1-mediated hematopoietic stem-cell emergence is controlled by a Gata/Ets/SCL-regulated enhancer. Blood 110 (2007) 4188-97.

[73] M. Hoogenkamp, H. Krysinska, R. Ingram, et al., The Pu.1 locus is differentially regulated at the level of chromatin structure and noncoding transcription by alternate mechanisms at distinct developmental stages of hematopoiesis. Molecular and cellular biology 27 (2007) 7425-38.

[74] N. Pencovich, R. Jaschek, A. Tanay, Y. Groner, Dynamic combinatorial interactions of RUNX1 and cooperating partners regulates megakaryocytic differentiation in cell line models. Blood 117 (2011) e1-14.

[75] M. Fowler, E. Borazanci, L. McGhee, et al., RUNX1 (AML-1) and RUNX2 (AML-3) cooperate with prostate-derived Ets factor to activate transcription from the PSA upstream regulatory region. Journal of cellular biochemistry 97 (2006) 1-17.

[76] M. Arman, N. Aguilera-Montilla, V. Mas, et al., The human CD6 gene is transcriptionally regulated by RUNX and Ets transcription factors in T cells. Molecular immunology 46 (2009) 2226-35.

[77] H. Huang, M. Yu, T.E. Akie, et al., Differentiation-dependent interactions between RUNX-1 and FLI-1 during megakaryocyte development. Molecular and cellular biology 29 (2009) 4103-15.

[78] K. Jackson Behan, J. Fair, S. Singh, et al., Alternative splicing removes an Ets interaction domain from Lozenge during Drosophila eye development. Development genes and evolution 215 (2005) 423-35.

[79] J. Marks-Bluth, J.E. Pimanda, Cell signalling pathways that mediate haematopoietic stem cell specification. The international journal of biochemistry \& cell biology 44 (2012) 2175-84.

[80] T.M. Schlaeger, H.K. Mikkola, C. Gekas, H.B. Helgadottir, S.H. Orkin, Tie2Cre-mediated gene ablation defines the stem-cell leukemia gene (SCL/tal1)-dependent window during hematopoietic stem-cell development. Blood 105 (2005) 3871-4.

[81] K.A. Dooley, A.J. Davidson, L.I. Zon, Zebrafish scl functions independently in hematopoietic and endothelial development. Developmental biology 277 (2005) 522-36.

[82] L.J. Patterson, M. Gering, R. Patient, Scl is required for dorsal aorta as well as blood formation in zebrafish embryos. Blood 105 (2005) 3502-11.

[83] J.E. Pimanda, K. Ottersbach, K. Knezevic, et al., Gata2, Fli1, and Scl form a recursively wired gene-regulatory circuit during early hematopoietic development. Proceedings of the National Academy of Sciences of the United States of America 104 (2007) 17692-7.

[84] M.J. Chen, T. Yokomizo, B.M. Zeigler, E. Dzierzak, N.A. Speck, Runx1 is required for the endothelial to haematopoietic cell transition but not thereafter. Nature 457 (2009) 887-91.

[85] T. Hendricks, N. Francis, D. Fyodorov, E.S. Deneris, The ETS domain factor Pet-1 is an early and precise marker of central serotonin neurons and interacts with a conserved element in serotonergic genes. The Journal of neuroscience : the official journal of the Society for Neuroscience 19 (1999) 10348-56.

[86] C. Lillesaar, B. Tannhauser, C. Stigloher, E. Kremmer, L. Bally-Cuif, The serotonergic phenotype is acquired by converging genetic mechanisms within the zebrafish central nervous system. Developmental dynamics : an official publication of the American Association of Anatomists 
236 (2007) 1072-84.

[87] Y.C. Wang, M.B. Zuraek, Y. Kosaka, et al., The ETS oncogene family transcription factor FEV identifies serotonin-producing cells in normal and neoplastic small intestine. Endocrine-related cancer 17 (2010) 283-91.

[88] L. Wang, T. Liu, L. Xu, et al., Fev regulates hematopoietic stem cell development via ERK signaling. Blood (2013).

[89] R.A. Nimmo, F.J. Slack, An elegant miRror: microRNAs in stem cells, developmental timing and cancer. Chromosoma 118 (2009) 405-18.

[90] U. Bissels, A. Bosio, W. Wagner, MicroRNAs are shaping the hematopoietic landscape. Haematologica 97 (2012) 160-7.

[91] H. Zhao, D. Wang, W. Du, D. Gu, R. Yang, MicroRNA and leukemia: tiny molecule, great function. Critical reviews in oncology/hematology 74 (2010) 149-55.

[92] H. Zhan, C. Cardozo, A. Raza, MicroRNAs in myeloproliferative neoplasms. British journal of haematology (2013).

[93] C.M. Sturgeon, L. Chicha, A. Ditadi, et al., Primitive erythropoiesis is regulated by miR-126 via nonhematopoietic Vcam-1+ cells. Developmental cell 23 (2012) 45-57.

[94] T.A. Harris, M. Yamakuchi, M. Kondo, P. Oettgen, C.J. Lowenstein, Ets-1 and Ets-2 regulate the expression of microRNA-126 in endothelial cells. Arteriosclerosis, thrombosis, and vascular biology 30 (2010) 1990-7.

[95] M.K. Lalwani, M. Sharma, A.R. Singh, et al., Reverse genetics screen in zebrafish identifies a role of miR-142a-3p in vascular development and integrity. PloS one 7 (2012) e52588.

[96] C.Z. Chen, L. Li, H.F. Lodish, D.P. Bartel, MicroRNAs modulate hematopoietic lineage differentiation. Science 303 (2004) 83-6.

[97] T. Nishiyama, R. Kaneda, T. Ono, et al., miR-142-3p is essential for hematopoiesis and affects cardiac cell fate in zebrafish. Biochemical and biophysical research communications 425 (2012) 755-61.

[98] Y. Sun, J. Sun, T. Tomomi, et al., PU.1-Dependent Transcriptional Regulation of miR-142 Contributes to Its Hematopoietic Cell-Specific Expression and Modulation of IL-6. Journal of immunology 190 (2013) 4005-13.

[99] S. Ghani, P. Riemke, J. Schonheit, et al., Macrophage development from HSCs requires PU.1-coordinated microRNA expression. Blood 118 (2011) 2275-84.

[100] X.S. Wang, J.N. Gong, J. Yu, et al., MicroRNA-29a and microRNA-142-3p are regulators of myeloid differentiation and acute myeloid leukemia. Blood 119 (2012) 4992-5004.

[101] S. Kayali, G. Giraud, F. Morle, B. Guyot, Spi-1, Fli-1 and Fli-3 (miR-17-92) oncogenes contribute to a single oncogenic network controlling cell proliferation in friend erythroleukemia. PloS one 7 (2012) e46799.

[102] V. Pospisil, K. Vargova, J. Kokavec, et al., Epigenetic silencing of the oncogenic miR-17-92 cluster during PU.1-directed macrophage differentiation. The EMBO journal 30 (2011) 4450-64.

[103] Y.C. Chan, S. Khanna, S. Roy, C.K. Sen, miR-200b targets Ets-1 and is down-regulated by hypoxia to induce angiogenic response of endothelial cells. The Journal of biological chemistry 286 (2011) 2047-56.

[104] Y.C. Chan, S. Roy, Y. Huang, S. Khanna, C.K. Sen, The microRNA miR-199a-5p down-regulation switches on wound angiogenesis by derepressing the v-ets erythroblastosis 
virus E26 oncogene homolog 1-matrix metalloproteinase-1 pathway. The Journal of biological chemistry 287 (2012) 41032-43.

[105] P. Romania, V. Lulli, E. Pelosi, et al., MicroRNA 155 modulates megakaryopoiesis at progenitor and precursor level by targeting Ets-1 and Meis1 transcription factors. British journal of haematology 143 (2008) 570-80.

[106] E. Coskun, E.K. von der Heide, C. Schlee, et al., The role of microRNA-196a and microRNA-196b as ERG regulators in acute myeloid leukemia and acute T-lymphoblastic leukemia. Leukemia research 35 (2011) 208-13. 


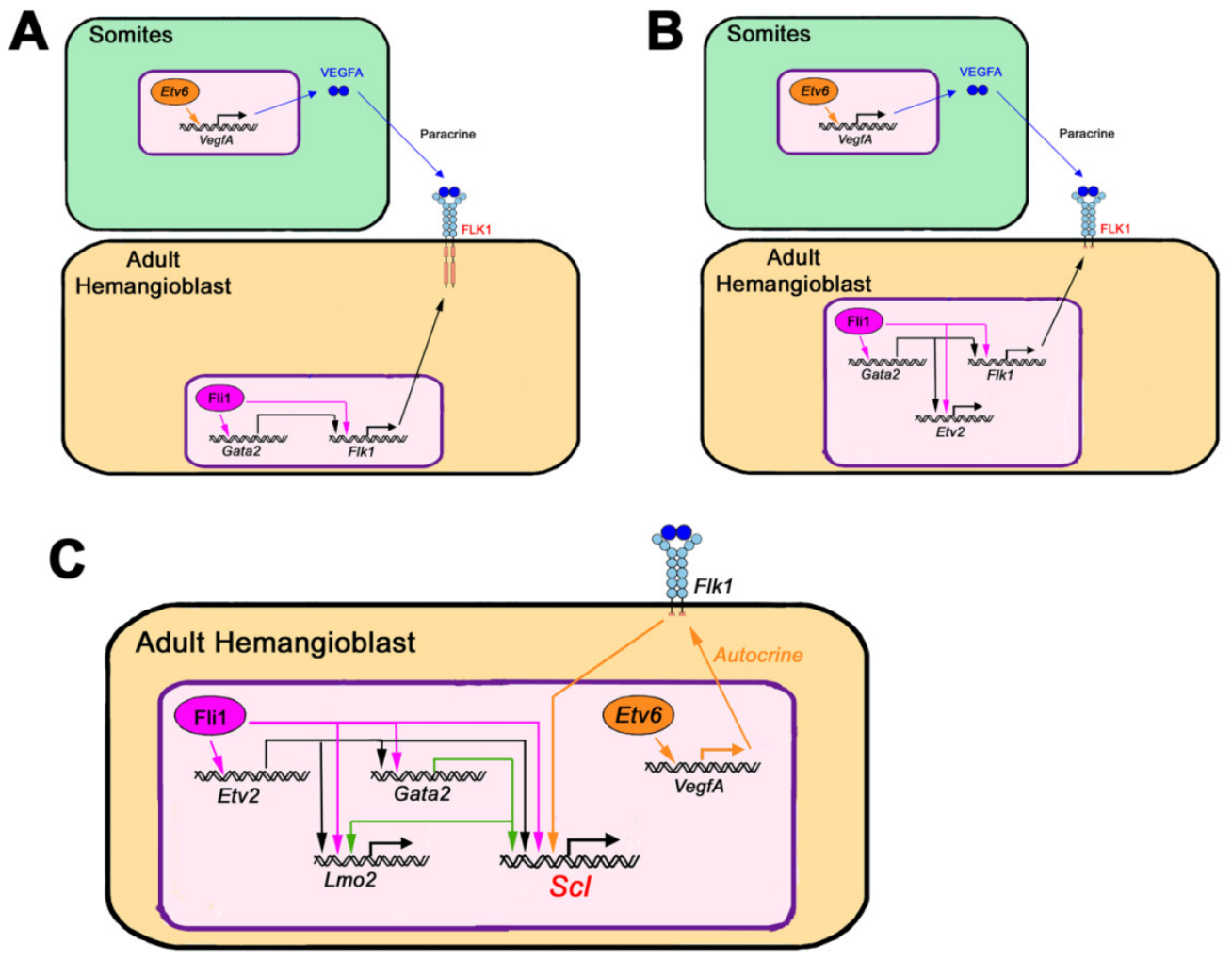

Figure 1. ETS TFs are at the core of the GRN programming adult hemangioblasts in the lateral plate mesoderm.

A. The adult hemangioblast program is initiated when VEGFA produced in the somites under the control of Etv6, activates its receptor, Flk1, in hemangioblasts precursors localized in the dorsal lateral plate mesoderm. Flk1 expression in hemangioblasts precursors is controlled by Fli1 and Gata2.

B. Fli1 and Gata2 activate Etv2 expression in adult hemangioblasts precursors independently of VEGFA signaling.

C. In a VEGFA-independent manner, Etv2 controls the expression of genes which synergize with cell autonomous VEGFA signaling to activate the expression of $\mathrm{Scl}$, a gene essential for adult hemangioblast determination. 


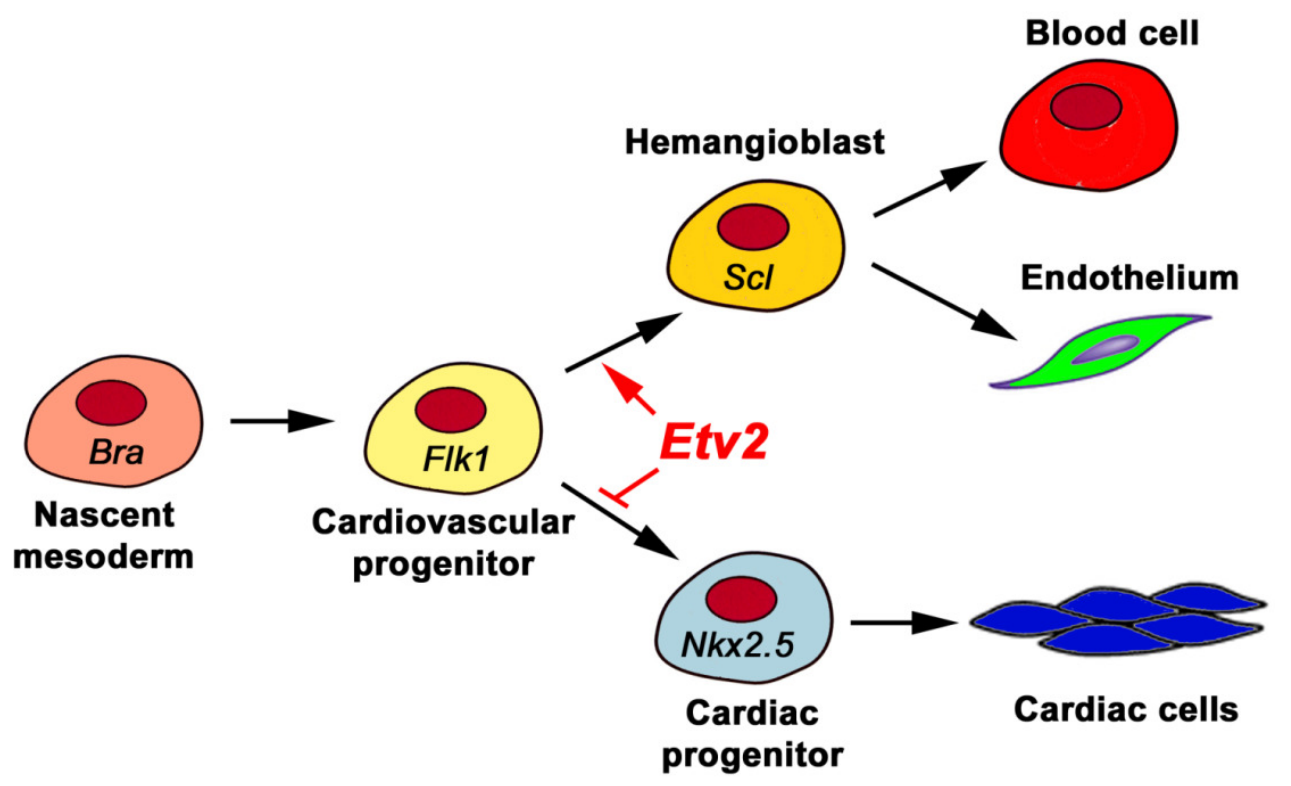

Figure 2. Etv2 controls the hematopoietic potential of nascent cardiovascular progenitors.

Expression of Etv2 in Flk1+ cardiovascular progenitors induces the hemangioblast program while repressing the cardiac fate. In the absence of Etv2 expression, Flk1+ cells differentiate into cardiac cells. 


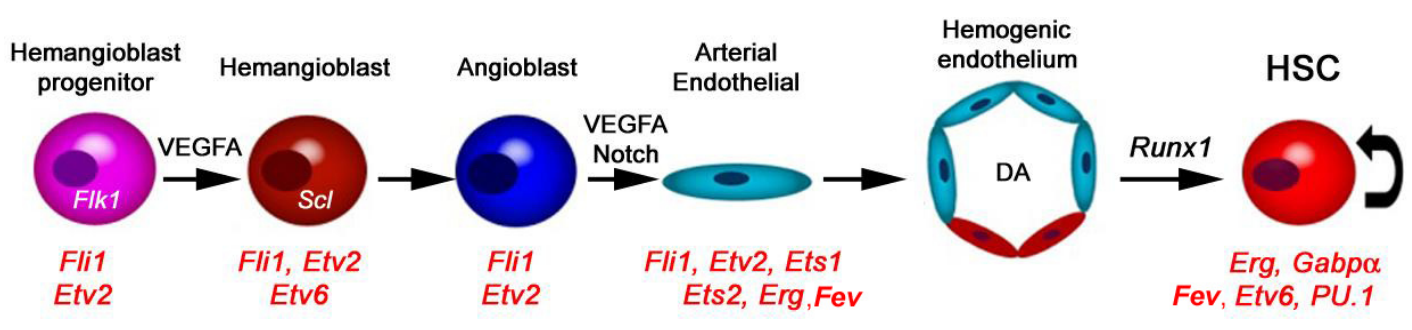

Figure 3. ETS transcription factors essential for the programming of the hematopoietic stem cell

lineage. Schematics showing the cellular hierarchy in the programming of HSCs in developing embryos. ETS TFs known to be critical for the development or maintenance of each cellular type are indicated. 